\title{
Reducing $\mathrm{CO}_{2}$ to $\mathrm{CO}$ and $\mathrm{H}_{2} \mathrm{O}$ on $\mathrm{Ni}(110)$ : the Influence of Subsurface Hydrogen
}

Wei Lin, ${ }^{\dagger}$ Kelsey M. Stocker,${ }^{\dagger}$ George C. Schatz*

Department of Chemistry, Northwestern University, Evanston, Illinois 60208, United States

\section{Corresponding Author}

*g-schatz@northwestern.edu

$\dagger$ These authors contributed equally 


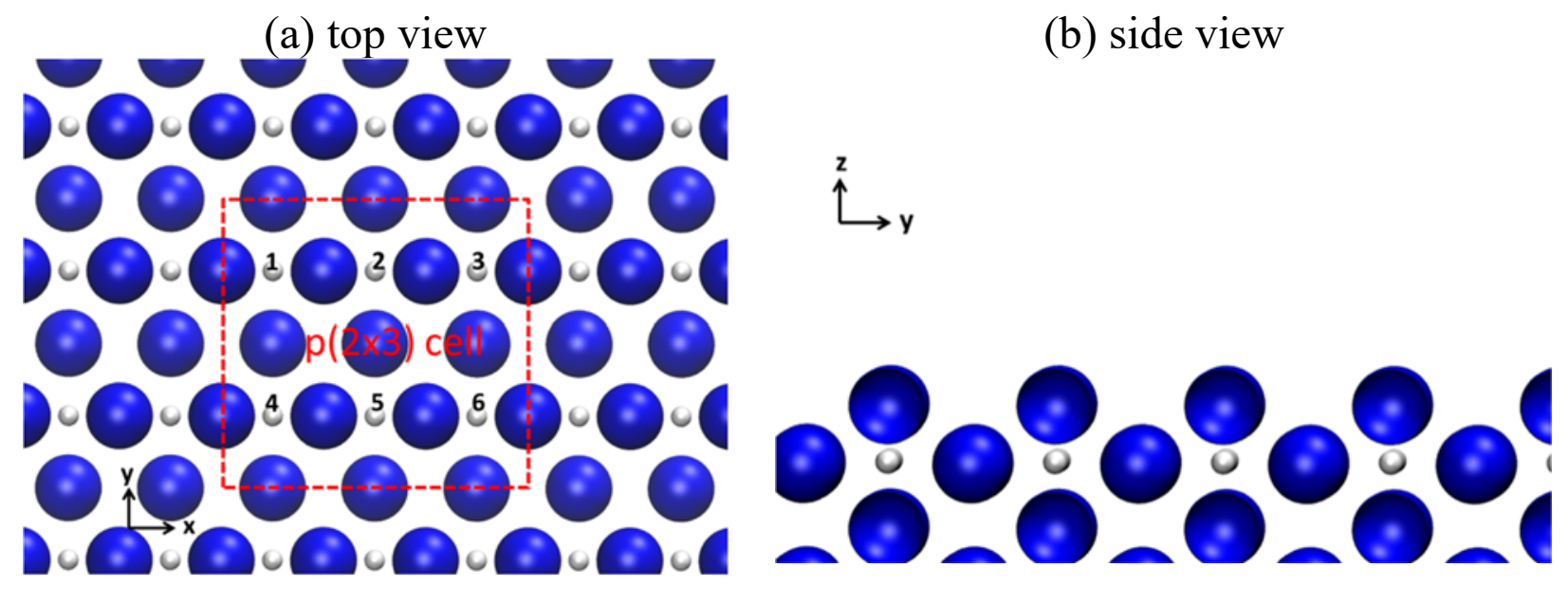

Figure S1. (a) Top and (b) side views of structures of hydrogen adsorption on octahedral sites (subsurface) of $\mathrm{Ni}(110)$ with 1 monolayer coverage. The $(2 \times 3)$ surface unit cell in a seven-layer slab used in this study is shown in the top view. In the unit cell, six hydrogen atoms of 1 monolayer coverage are labeled. 


$$
\text { a). } \begin{aligned}
E_{\text {ads }} & =-0.45 \mathrm{eV} \\
q & =-0.80|\mathrm{e}|
\end{aligned}
$$
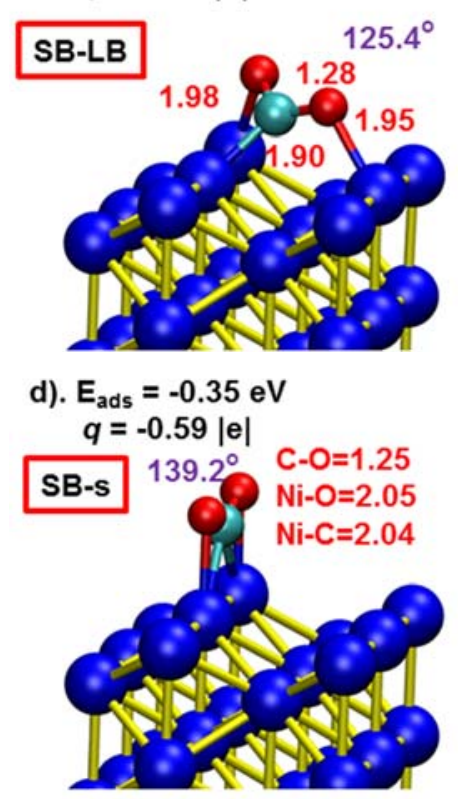

g). $E_{\text {ads }}=-0.27 \mathrm{eV}$

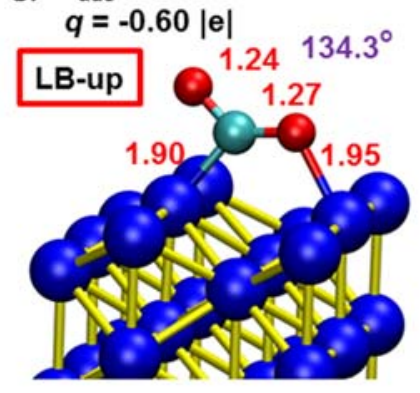

b). $E_{\text {ads }}=-0.42 \mathrm{eV}$

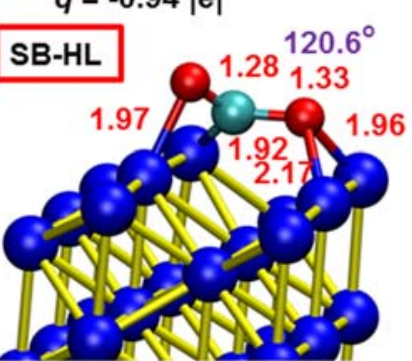

e). $E_{\text {ads }}=-0.33 \mathrm{eV}$

$$
q=-0.86|\mathrm{e}|
$$

$\mathrm{HL}$

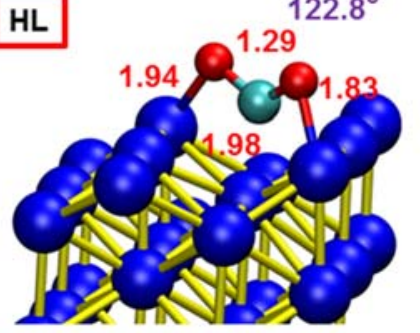

c). $E_{\text {ads }}=-0.39 \mathrm{eV}$

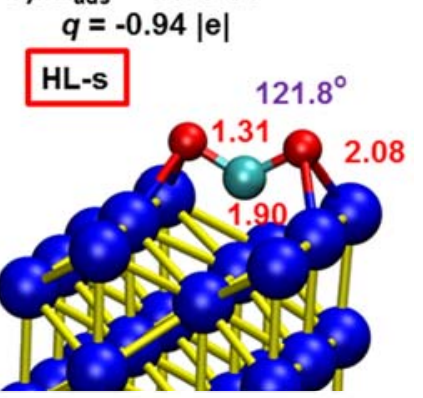

f). $E_{\text {ads }}=-0.28 \mathrm{eV}$

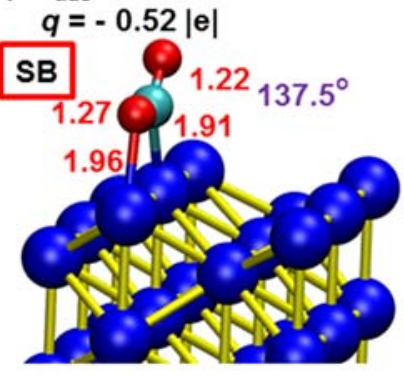

Figure S2. Structures, adsorption energies and charges of $\mathrm{CO}_{2}$ chemisorbed on a $\mathrm{Ni}(110)$ surface with 1/6 monolayer coverage. Values highlighted in red and purple are bond distances in $\AA$ and $\mathrm{O}-\mathrm{C}-\mathrm{O}$ bond angles. The definitions of the configurations are as follows: LB = long bridge; LB$\mathrm{s}=$ long bridge symmetric; HL-s = Hollow symmetric; SB-s = Short bridge symmetric; HL = Hollow; $\mathrm{SB}=$ Short bridge; LB-up = Long bridge up. 


$$
\begin{gathered}
\text { a). } \mathrm{E}_{\mathrm{ads}}=-0.65 \mathrm{eV} \\
q=-0.79|\mathrm{e}|
\end{gathered}
$$

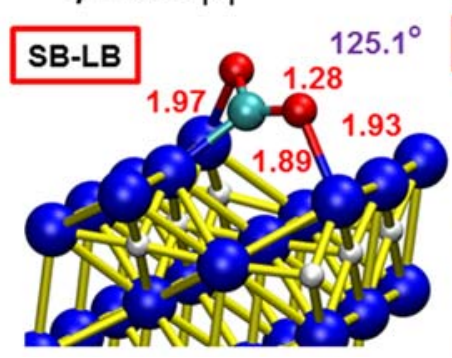

d). $E_{\text {ads }}=-0.53 \mathrm{eV}$

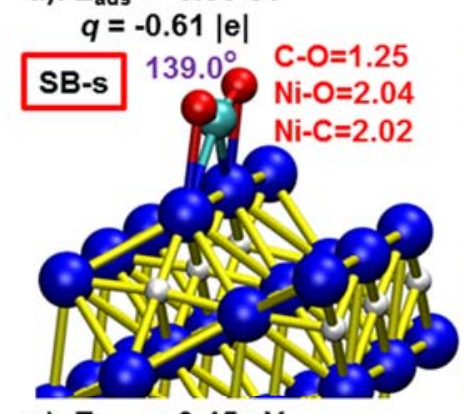

g). $E_{\text {ads }}=-0.45 \mathrm{eV}$

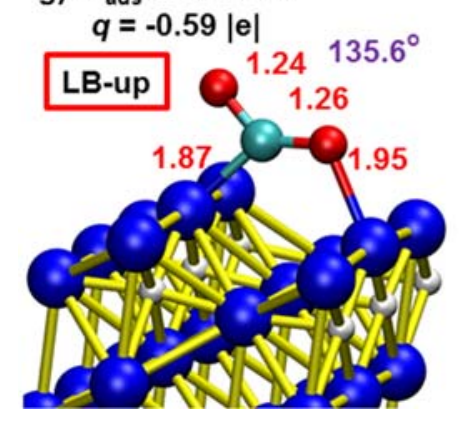

b). $E_{\text {ads }}=-0.672 \mathrm{eV}$

$q=-0.92|\mathrm{e}|$

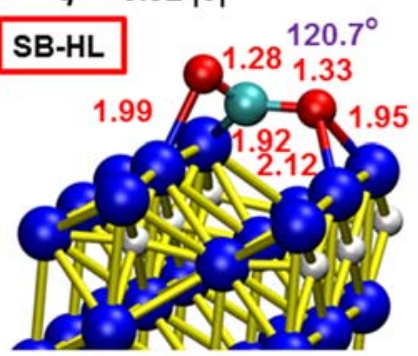

e). $E_{\text {ads }}=-0.62 \mathrm{eV}$

$q=-0.89|\mathrm{e}|$

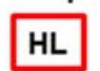

$\mathrm{HL}$

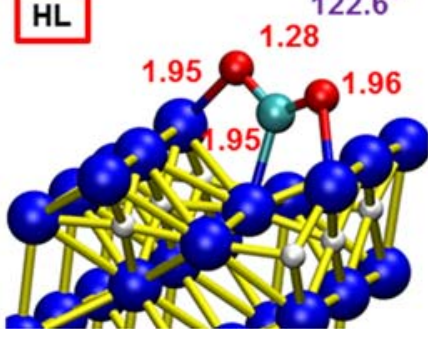

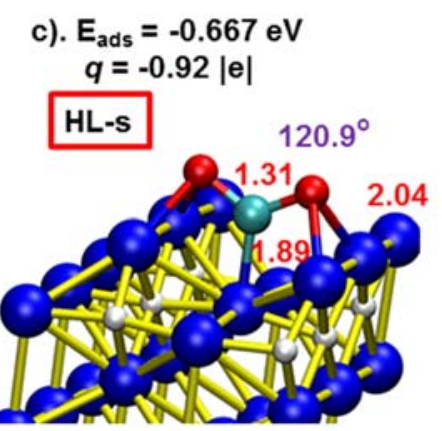

f). $E_{\text {ads }}=-0.42 \mathrm{eV}$

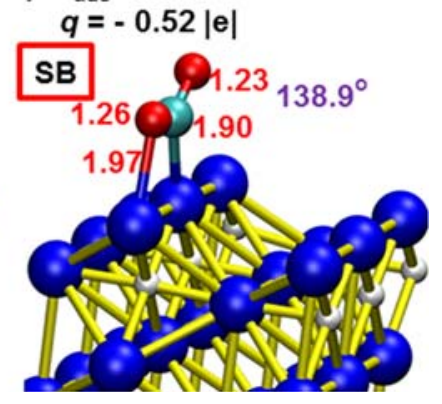

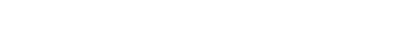
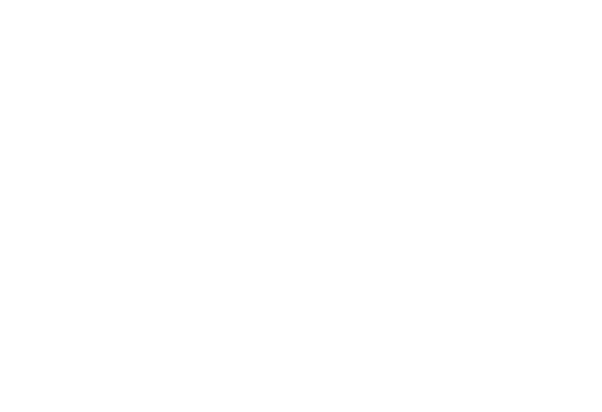

Figure S3. Structures, adsorption energies and charges of $\mathrm{CO}_{2}$ chemisorbed on $\mathrm{Ni}(110)$ with 1 monolayer coverage of subsurface hydrogen. Values highlighted in red and purple are bond distances in $\AA$ and O-C-O bond angles. Definitions of the configurations are as follows: $\mathrm{LB}=$ long bridge; LB-s = long bridge symmetric; HL-s = Hollow symmetric; SB-s = Short bridge symmetric; $\mathrm{HL}=$ Hollow; $\mathrm{SB}=$ Short bridge; LB-up = Long bridge up. 
(a) Top view

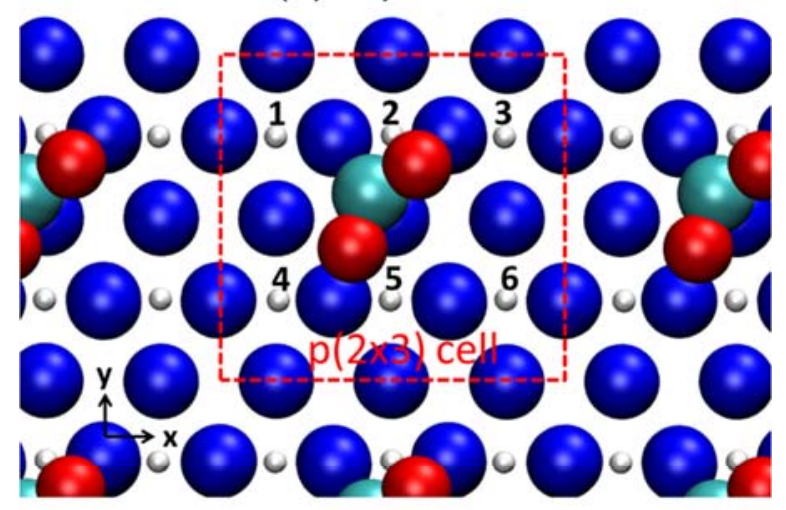

(b) Side view

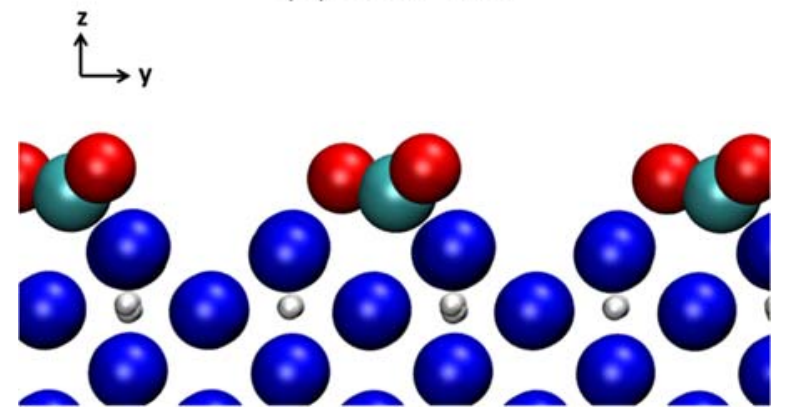

Figure S4. (a) Top and (b) side views of $\mathrm{CO}_{2}$ adsorption on $\mathrm{Ni}(110)$ in the presence of 1 monolayer subsurface hydrogen in octahedral sites $\left(\mathrm{H}_{\mathrm{b}}\right)$. The $(2 \times 3)$ surface unit cell in a sevenlayer slab used in current study is shown in top view. In the unit cell, six hydrogen atoms of 1 monolayer coverage are labeled. 
Table S1. The chemisorption energies $\left(E_{\text {ads }}, \mathrm{eV}\right)$ of subsurface hydrogen $\left(\mathrm{H}_{\text {sub }}\right)$ in $1^{\text {st }}$ layer of $\mathrm{O}_{\mathrm{h}}$ sites with all possible configurations from 1/6 to $1 \mathrm{ML}$ coverage. The locations of $\mathrm{H}_{\text {sub }}$ are labeled in Figure $\mathrm{S} 1$. The $E_{\text {ads }}$ per $\mathrm{H}_{\text {sub }}$ (in $\mathrm{eV}$ ) shows the adsorption energies of each $\mathrm{H}_{\text {sub }}$ within all range of coverages.

\begin{tabular}{|c|c|c|c|}
\hline No. of $\mathrm{H}_{\text {sub }}$ & Location of $\mathrm{H}_{\text {sub }}$ & $E_{\text {ads }}(\mathrm{eV})$ & $E_{\text {ads }}$ per $\mathrm{H}_{\text {sub }}(\mathrm{eV})$ \\
\hline 1 & & -2.13 & -2.13 \\
\hline 2 & $(1,5)$ & -4.25 & -2.12 \\
\hline & $(1,2)$ & -4.29 & -2.14 \\
\hline & $(1,4)$ & -4.28 & -2.14 \\
\hline 3 & $(1,2,3)$ & -6.51 & -2.17 \\
\hline & $(1,2,4)$ & -6.43 & -2.14 \\
\hline & $(1,2,6)$ & -6.41 & -2.14 \\
\hline 4 & $(1,2,4,5)$ & -8.61 & -2.15 \\
\hline & $(1,2,4,6)$ & -8.60 & -2.15 \\
\hline & $(1,2,3,4)$ & -8.65 & -2.16 \\
\hline 5 & & -10.82 & -2.16 \\
\hline 6 & & -13.04 & -2.17 \\
\hline
\end{tabular}

Table S2. Chemisorption energies $\left(E_{\text {ads, }}, e V\right)$ of subsurface/bulk hydrogen in $\mathrm{O}_{\mathrm{h}}$ and/or $\mathrm{T}_{\mathrm{d}}$ sites. Adsorption energy values are given per $\mathrm{H}$ atom.

\begin{tabular}{|c|c|}
\hline Coverage & $E_{\text {ads }}$ per $\mathrm{H}(\mathrm{eV})$ \\
\hline $1^{\text {st }} \mathrm{O}_{\mathrm{h}}$ layer, $1 \mathrm{ML}$ & -2.17 \\
\hline $2^{\text {nd }} \mathrm{O}_{\mathrm{h}}$ layer, 1 ML & -2.21 \\
\hline $3^{\text {rd }} \mathrm{O}_{\mathrm{h}}$ layer, 1 ML & -2.21 \\
\hline $\mathrm{T}_{\mathrm{d}}$ layer, 2 ML & -1.99 \\
\hline $\begin{array}{c}1^{\text {st }} \mathrm{O}_{\mathrm{h}} \text { layer, } 1 \mathrm{ML}+2^{\text {nd }} \\
\mathrm{O}_{\mathrm{h}} \text { layer, } 1 \mathrm{ML}\end{array}$ & -2.13 \\
\hline $\begin{array}{c}1^{\text {st }} \mathrm{O}_{\mathrm{h}} \text { layer, } 1 \mathrm{ML}+\mathrm{T}_{\mathrm{d}} \\
\text { layer, } 2 \mathrm{ML}\end{array}$ & -2.06 \\
\hline
\end{tabular}


Table S3. Chemisorption energies $\left(E_{\mathrm{ads}}, \mathrm{eV}\right)$ of $\mathrm{CO}_{2}$ on $\mathrm{Ni}(110)$ surface. Structures of adsorption sites are shown in Figure S2. Values in 'Diff' are the differences in chemisorption energies between the current work and Ref. S1, in which 2 x 2 unit cell was used. In both of our work and Ref. S2, a 2 x 3 unit cell was used.

\begin{tabular}{|c|c|c|c|c|}
\hline Ads. site & $E_{\text {ads }}(\mathrm{eV})$ & Ref. S1 & Diff $(\mathrm{eV})$ & Ref. S2 \\
\hline SB-LB & -0.45 & -0.39 & -0.06 & -0.47 \\
\hline HL-s & -0.39 & -0.18 & -0.21 & $/$ \\
\hline SB-s & -0.35 & -0.23 & -0.12 & $/$ \\
\hline HL & -0.33 & -0.14 & -0.19 & $/$ \\
\hline SB & -0.28 & -0.21 & -0.07 & $/$ \\
\hline LB-up & -0.27 & -0.22 & -0.05 & $/$ \\
\hline
\end{tabular}

Table S4. Chemisorption energies $\left(E_{\text {ads, }}, \mathrm{eV}\right)$ of $\mathrm{CO}_{2}$ on $\mathrm{Ni}(110)$ with subsurface hydrogen $\left(\mathrm{H}_{\text {sub }}\right)$ coverages up to 1 monolayer. The locations of $\mathrm{H}_{\text {sub }}$ are labeled in Figure S4a. Diff means the energy difference between $E_{\text {ads }}\left(\# \mathrm{H}_{\text {sub }}\right)$ and $E_{\text {ads }}\left(0 \mathrm{H}_{\text {sub }}\right)$.

\begin{tabular}{|c|c|c|c|}
\hline No. of $\mathrm{H}_{\text {sub }}$ & Location of $\mathrm{H}_{\text {sub }}$ & $E_{\text {ads }}(\mathrm{eV})$ & Diff \\
\hline 0 & & -0.45 & $/$ \\
\hline 1 & $(1)$ or $(6)$ & -0.45 & 0.00 \\
\hline & $(2)$ or $(5)$ & -0.50 & -0.05 \\
\hline & $(3)$ & -0.54 & -0.09 \\
\hline & $(4)$ & -0.52 & -0.07 \\
\hline 2 & $(2,3)$ & -0.58 & -0.13 \\
\hline & $(2,5)$ & -0.54 & -0.09 \\
\hline & $(2,4)$ & -0.57 & -0.12 \\
\hline & $(3,6)$ & -0.51 & -0.06 \\
\hline 3 & $(1,2,3)$ & -0.57 & -0.12 \\
\hline & $(1,2,5)$ & -0.54 & -0.09 \\
\hline & $(2,3,4)$ & -0.65 & -0.20 \\
\hline & $(1,3,6)$ & -0.53 & -0.08 \\
\hline 4 & $(1,2,3,5)$ & -0.62 & -0.17 \\
\hline & $(1,2,4,5)$ & -0.59 & -0.14 \\
\hline & $(2,3,4,5)$ & -0.68 & -0.23 \\
\hline & $(1,3,5,6)$ & -0.52 & -0.07 \\
\hline 5 & $(1,2,3,4,5)$ & -0.66 & -0.21 \\
\hline & $(1,3,4,5,6)$ & -0.66 & -0.21 \\
\hline 6 & $(1,2,3,4,5,6)$ & -0.65 & -0.20 \\
\hline
\end{tabular}


Table S5. Effect of $1 \mathrm{ML}$ of subsurface hydrogen in top $\mathrm{O}_{\mathrm{h}}$ sites on vibrational frequencies of adsorbed $\mathrm{CO}_{2}$. Frequencies are given as [no $\mathrm{H}_{\text {sub }}$ / [with $\mathrm{H}_{\text {sub }}$ in $\mathrm{meV}$.

\begin{tabular}{|c|c|c|c|c|c|}
\hline Ads. site & Asymm. str. & Symm. str. & Bend & Hind. rot. & Ext. stretch \\
\hline Phys. & $291 / 291$ & $163 / 163$ & $78 / 77$ & $77 / 76$ & \\
\hline SB-LB & $174 / 175$ & $138 / 140$ & $88 / 89$ & $50 / 53$ & $44 / 46$ \\
\hline SB & $216 / 215$ & $139 / 142$ & $85 / 85$ & $62 / 63$ & $46 / 47$ \\
\hline SB-s & $218 / 215$ & $143 / 143$ & $80 / 82$ & $62 / 64$ & $43 / 45$ \\
\hline LB-up & $206 / 206$ & $141 / 142$ & $85 / 85$ & $57 / 57$ & $43 / 45$ \\
\hline HL-s & $164 / 161$ & $136 / 136$ & $85 / 86$ & $50 / 52$ & $47 / 48$ \\
\hline HL & $174 / 170$ & $137 / 135$ & $90 / 88$ & $60 / 47$ & $45 / 46$ \\
\hline SB-HL & $166 / 171$ & $126 / 126$ & $87 / 87$ & $47 / 48$ & $44 / 45$ \\
\hline
\end{tabular}

References:

(S1) Wang, S.-G.; Cao, D.-B.; Li, Y.-W.; Wang, J.; Jiao, H. J. Phys. Chem. B, 2005, 109, 18956.

(S2) Peng, G.; Sibener, S. J.; Schatz, G. C.; Mavrikakis, M. Surf. Sci., 2012, 606, 1055. 